Pacific Journal of Mathematics

MATCHED PAIRS OF LIE GROUPS ASSOCIATED TO
SOLUTIONS OF THE YANG-BAXTER EQUATIONS 


\title{
MATCHED PAIRS OF LIE GROUPS ASSOCIATED TO SOLUTIONS OF THE YANG-BAXTER EQUATIONS
}

\author{
SHAHN MAJid
}

Two groups $G, H$ are said to be a matched pair if they act on each other and these actions, $(\alpha, \beta)$, obey a certain compatibility condition. In such a situation one may form a bicrossproduct group, denoted $G_{\beta} \bowtie_{\alpha} H$. Also in this situation one may form a bicrossproduct Hopf, Hopf-von Neumann or Kac algebra obtained by simultaneous cross product and cross coproduct.

We show that every compact semi-simple simply-connected Lie group $G$ is a member of a matched pair, denoted $\left(G, G^{\star}\right)$, in a natural way.

As an example we construct the matched pair in detail in the case $\left(\mathrm{SU}(2), \mathrm{SU}(2)^{\star}\right)$ where

$$
\mathrm{SU}(2)^{\star}=\left\{\left(\begin{array}{cc}
x & 0 \\
z & x^{-1}
\end{array}\right): x \in \mathbf{R}_{>0}, z \in \mathbf{C}\right\}
$$

is the simply-connected group of a Lie algebra $\operatorname{su}(2)^{\star}$. Here $\operatorname{su}(2)^{\star}$ is defined with respect to a standard canonical solution of the CYBE on the complexification of $\mathrm{su}(2)$.

1. Introduction and preliminaries. The notion of a matched pair of groups was studied in [18] and more recently in [12]. Two groups $G$, $H$ are a matched pair if they act on each other and these actions $\alpha, \beta$ obey the condition

$$
\begin{gathered}
\forall x, y \in G, \mathscr{L}, \mathscr{M} \in H, \quad \alpha_{x^{-1}}(e)=e, \quad \beta_{\mathscr{L}^{-1}}(e)=e, \\
\alpha_{x^{-1}}(\mathscr{L} \mathscr{M})=\alpha_{x^{-1}}(\mathscr{L}) \alpha_{\beta_{\mathscr{L}^{-1}}(x)^{-1}}(\mathscr{M}), \\
\beta_{\mathscr{L}^{-1}}(x y)=\beta_{\mathscr{L}^{-1}}(x) \beta_{\alpha_{x^{-1}}(\mathscr{L})^{-1}}(y)
\end{gathered}
$$

where $e$ denotes the relevant group identity. We have chosen here conventions in which the natural objects are right actions $\alpha_{x^{-1}}, \beta_{\mathscr{L}^{-1}}$. Right action conventions are the natural choice for actions on manifolds, cf. [7, Chapter $1, \S 4$ ]. There are also equivalent versions of $(*)$ suitable for left actions and for one left action and one right action [12, Example 3.14]. In this case both [18] and [12] noted that one may form a bicrossproduct group $G \bowtie H$ defined by

$$
\begin{aligned}
(x, \mathscr{L}) \cdot(y, \mathscr{M}) & =\left(\beta_{\mathscr{M}^{-1}}\left(x^{-1}\right)^{-1} y, \mathscr{L} \alpha_{x}(\mathscr{M})\right), \\
(x, \mathscr{L})^{-1} & =\left(\beta_{\mathscr{L}}(x)^{-1}, \alpha_{x^{-1}}\left(\mathscr{L}^{-1}\right)\right),
\end{aligned}
$$


and in the case when $G$ and $H$ are finite, a bicrossproduct Hopf algebra $k[G] \bowtie k(H)$. Here $k$ is a field, $k[G]$ the convolution algebra on $G$ and $k(H)$ the function algebra on $H$. [18] was able to show, in the context of algebraic groups, that a certain Hopf algebra of Taft and Wilson was of this form. If $G$ is Abelian and $\alpha$ trivial then the bicrossproduct Hopf algebra $k[G] \bowtie k(H)$ is isomorphic to $k(\hat{G} \bowtie H)$, but the bicrossproduct makes sense even when $G$ is non-Abelian, and even when $\alpha$ is non-trivial. In this latter case the bicrossproduct Hopf algebra is non-commutative and therefore cannot be the algebra of functions on any space. This is the point of view relevant to noncommutative geometry [3], [16]. New examples in this latter context were given in [12]. These new examples were motivated from physical considerations [13].

In order to generalize these results to the category of Lie groups, to yield bicrossproduct Lie groups and (in principle) bicrossproduct Hopf-von Neumann algebras, one must have examples of matched pairs of Lie groups. This is particularly interesting because the corresponding Lie algebra version of the above Hopf algebras, in which the role of $k[G]$ is played by $U(g)$, the universal enveloping algebra, remains an open problem. This would therefore appear to be a construction in which topology and algebra play an interdependent role.

Hopf algebras have been connected in a very different context with the Yang-Baxter Equations (YBE). It turns out that this connection is relevant also to the bicrossproduct construction, see [12, §4]. In [12, Example 4.2] it is shown that at the Lie algebra level, a pair of finitedimensional Lie algebras of the form $\left(g, g^{*}\right)$ with the coadjoint actions are a matched pair iff $g$ is a Lie bialgebra in the sense introduced by Drinfel'd [4] in connection with the Classical Yang-Baxter Equations (CYBE). This will be developed further in $\S 2$ below. By exploiting this, we are able in this paper to give a general constructive method that canonically associates to a suitable Lie group $G$ and a suitable solution of the CYBE on its complexified Lie algebra, a certain Lie group, the "Yang-Baxter-matching group", denoted $G^{\star}$, forming a matched pair $\left(G, G^{\star}\right)$. (Previously, solutions of the CYBE have been connected to the existence of a Poisson structure on $G$ (and the product Poisson structure on $G \times G$ ) such that the group product is Poisson [4], but this point of view will not be used here.) The method is carried out in detail for the case $G=\mathrm{SU}(2)$ and the group actions computed explicitly. The main results are Theorem 4.2 and Theorem 4.3, along with 
Lemma 4.1 and Corollary 4.6. The corresponding Hopf-von Neumann algebras will be presented in [14].

All Lie groups are assumed connected with their standard differential structure. $\xi, \xi^{\prime}$ will always denote elements of a finite dimensional Lie algebra $g$ or its complexification $g_{\mathrm{C}}, l, l^{\prime}$ of a finite dimensional Lie algebra $h$ or its complexification, $x, y$ of Lie group $G$ and $\mathscr{L}, \mathscr{L}^{\prime}$, $\mathscr{M}$ of a Lie group $H . L_{x}(y)=x y$ and $R_{x}(y)=y x$ denote left and right group translations. $\langle$,$\rangle denotes pairing of a space with its dual space$ and an unused argument denotes an element of a dual space. We work over $\mathbf{R}$ or $\mathbf{C}$. The linear dual of $g$ is denoted $g^{*}$. This should not be confused with $g^{\star}$ which denotes a certain real form of $g_{\mathbf{C}}^{*}$. All maps are required to be at least continuous.

2. Canonical solution of the CYBE on $g_{\mathrm{C}}$, the construction of $h=g^{\star}$ and Lie algebra actions, $\alpha$ and $\beta$. Let $g$ be a finite dimensional Lie algebra. Drinfel'd [4] and co-workers showed that if $r: g^{*} \rightarrow g$ is a linear map and $\alpha: g \otimes g^{*} \rightarrow g^{*}$ is defined by dualizing the Lie bracket on $g,\left\langle\alpha_{\xi}(l), \xi^{\prime}\right\rangle=\left\langle l,\left[\xi^{\prime}, \xi\right]\right\rangle$ for all $\xi_{,}, \xi^{\prime} \in g, l \in g^{*}$ (i.e., the coadjoint action of $g$ on $\left.g^{*}\right)$, then the bracket on $h=g^{*}$ defined by $\left[l, l^{\prime}\right]=$ $\alpha_{r(l)}\left(l^{\prime}\right)+\alpha_{r^{*}\left(l^{\prime}\right)}(l)$ is a Lie algebra structure if $r([])-,[r(), r()] \in$ $g \otimes g \otimes g$ is invariant under the adjoint action of $g$ and if $r=-r^{*}$ is skew-adjoint (i.e. $\left\langle r(l), l^{\prime}\right\rangle=-\left\langle l, r\left(l^{\prime}\right)\right\rangle$ for all $\left.l, l^{\prime} \in g^{*}\right)$. Moreover, in this case we showed in $[12, \S 4]$ that if $\beta: h \otimes g \rightarrow g$ is defined by dualizing this Lie bracket, $\left\langle\beta_{l}(\xi), l^{\prime}\right\rangle=\left\langle\xi,\left[l^{\prime}, l\right]\right\rangle$ (i.e., the coadjoint action of $g^{*}$ on $\left.g\right)$, then $(g, h, \alpha, \beta)$ are a matched pair of Lie algebras. This means that $\alpha$ is a linear representation of $g$ on $h$ and $\beta$ is a linear representation of $h$ on $g$ and these obey the compatibility condition

$(* *)$

$$
\begin{gathered}
\forall \xi, \xi^{\prime} \in g, l, l^{\prime} \in h, \\
\alpha_{\xi}\left(\left[l, l^{\prime}\right]\right)=\left[\alpha_{\xi}(l), l^{\prime}\right]+\left[l, \alpha_{\xi}\left(l^{\prime}\right)\right]+\alpha_{-\beta_{l}(\xi)}\left(l^{\prime}\right)-\alpha_{-\beta_{l^{\prime}}(\xi)}(l), \\
\beta_{l}\left(\left[\xi, \xi^{\prime}\right]\right)=\left[\beta_{l}(\xi), \xi^{\prime}\right]+\left[\xi, \beta_{l}\left(\xi^{\prime}\right)\right]+\beta_{-\alpha_{\xi}(l)}\left(\xi^{\prime}\right)-\beta_{-\alpha_{\xi^{\prime}}(l)}(\xi) .
\end{gathered}
$$

The case $r([])-,[r(), r()]$ vanishing is known as the CYBE and when $g$ is simple with inverse Killing form $K^{-1}: g^{*} \rightarrow g$ then the case

$$
r([,])-[r(), r()]=\left[K^{-1}(), K^{-1}()\right]
$$

is known as the Modified CYBE [17, Eqn. (12)]. (A suitable factor can also be included on the right hand side, but this can be absorbed in a rescaling of $r$ as observed in [17].) In a general setting, many solutions of the CYBE and MCYBE are known [1]. According to [6, Prop. 2.1], the MCYBE has at least one non-trivial solution that holds 
for all simple complex Lie algebras. If $\{\lambda\}$ is an ordered root system for $g$ with Chevalley-Weyl root vectors $\left\{E_{\lambda}\right\}$ then this solution is

$$
r=\sum_{\lambda} \frac{E_{\lambda} \otimes\left\langle E_{-\lambda,}\right\rangle \operatorname{sgn}(\lambda)}{K\left(E_{\lambda}, E_{-\lambda}\right)}+K^{-1} t .
$$

At present, $t=0$.

When $g$ is a real Lie algebra, one may first solve the MCYBE as above for $g_{C}$ the complexified Lie algebra and then attempt restriction of the resulting $g_{\mathrm{C}}^{*}$ to a suitable real form, which we denote $g^{\star}$, in such a way that $g$ and $h=g^{\star}$ are a matched pair of real Lie algebras. Because $K$ is ad-invariant and symmetric, the addition of the $t K^{-1}$ term shown does not change the bracket on $g_{\mathrm{C}}^{*}$ or $h$ as defined above, but results in $r([])-,[r(), r()]=\left(1-t^{2}\right)\left[K^{-1}(), K^{-1}()\right]$ so that the case $t=1$ (or -1 ) provides a useful homomorphism of $h$ to $g_{C}$. Under favorable circumstances, this is an embedding so that both $g, h$ can be viewed as subalgebras of $g_{C}$.

Proposition 2.1. Let $g$ be a compact real simple Lie algebra with complexification $g_{\mathrm{C}}$. Choose a Cartan subalgebra and root vectors $\left\{H_{\lambda}, E_{\lambda}\right\}$ such that $g$ is the compact real form of $g_{C}$ defined in [5, Chapter III, Thm. 6.3], and let $r$ be the solution, equation (1), of the MCYBE on $g_{\mathrm{C}}$ in this basis, so that $g_{\mathrm{C}}^{*}$ is a Lie algebra. Define

$$
g^{\star}=\imath K(g,)
$$

where $K$ denotes the Killing form on $g_{\mathbf{C}}$. Then $g^{\star}$ is a real form of $g_{\mathbf{C}}^{*}$ and the mutual co-adjoint actions of the matched pair $\left(g_{\mathbf{C}}, g_{\mathbf{C}}^{*}\right)$ restrict to a matched pair $\left(g, g^{\star}\right)$. Furthermore, $r: g^{\star} \rightarrow g_{\mathrm{C}}$ at $t=1$ is an inclusion.

Proof. Let $\left\{H_{\lambda}, E_{\lambda}\right\}$ be a Chevalley-Weyl basis of $g_{\mathbf{C}}$ normalized so that $K\left(E_{\lambda}, E_{-\lambda}\right)=1$ and $K\left(H, H_{\lambda}\right)=\lambda(H)$ for $H$ in the Cartan subalgebra and root $\lambda$ [5, Chapter III, Thm. 5.6]. Let $u$ be the compact real form defined in [5, Chapter III, Thm. 6.3]. Then there is an inner automorphism $\psi$ of $g_{\mathbf{C}}$ (connected to the identity) such that $g=$ $\psi(u)$ [5, Chapter III, Cor. 7.3]. Hence applying $\psi$ to the generators $\left\{H_{\lambda}, E_{\lambda}\right\}$ we obtain a new basis with the same normalization such that the compact real form constructed in this basis is just $g$. Hence without loss of generality we assume now $u=g$, i.e. explicitly

$$
\begin{aligned}
& g=\operatorname{span}_{\mathbf{R}}\left\{X_{\lambda}, Y_{\lambda}, Z_{\lambda}\right\}, \\
& \quad \text { where } X_{\lambda}=E_{\lambda}-E_{-\lambda}, \quad Y_{\lambda}=\imath\left(E_{\lambda}+E_{-\lambda}\right), \quad Z_{\lambda}=\imath H_{\lambda} .
\end{aligned}
$$


According to our definition, $g^{\star}$ is then the real span of $\bar{X}_{\lambda}=\imath K\left(X_{\lambda},\right)$, $\bar{Y}_{\lambda}=\imath K\left(Y_{\lambda},\right), \bar{Z}_{\lambda}=\imath K\left(Z_{\lambda},\right)$ and we compute

$$
r\left(\bar{X}_{\lambda}\right)=\operatorname{sgn}(\lambda) Y_{\lambda}+t t X_{\lambda}, \quad r\left(\bar{Y}_{\lambda}\right)=-\operatorname{sgn}(\lambda) X_{\lambda}+\imath t Y_{\lambda}, \quad r\left(\bar{Z}_{\lambda}\right)=\imath t Z_{\lambda},
$$

so that $r$ is an inclusion at $t=1$. To compute the Lie algebra structure it is convenient to work with $t=0$. In that case $r(l)$ lies in $g$ for all $l \in g^{\star}$. For all $\xi \in g, \xi^{\prime \prime} \in g_{\mathrm{C}}$, and $l^{\prime}=\imath K\left(\xi^{\prime},\right) \in g^{\star}$ where $\xi^{\prime} \in g$, we have $\left\langle\alpha_{\xi}\left(l^{\prime}\right), \xi^{\prime \prime}\right\rangle=\imath K\left(\xi^{\prime},\left[\xi^{\prime \prime}, \xi\right]\right)=\imath K\left(\left[\xi, \xi^{\prime}\right], \xi^{\prime \prime}\right)$ by ad-invariance of $K$, so that the co-adjoint action $\alpha$ restricts to an action of $g$ on $g^{\star}$. Hence the bracket $\left[l, l^{\prime}\right]=\alpha_{r(l)}\left(l^{\prime}\right)-\alpha_{r\left(l^{\prime}\right)}(l)$ also restricts to $g^{\star}$ (here $r$ is skew at $t=0)$. Like-wise, in terms of $\alpha, \beta_{l}(\xi)=r \circ \alpha_{\xi}(l)+[r(l), \xi]$ also restricts. This completes the proof. (It is also necessary to verify that equation (1) at $t=0$ does indeed solve the MCYBE on $g_{C}$ as stated in [6, Prop. 2.1]. The first step is the re-arrangement

$$
\begin{aligned}
& \left\langle l, r\left(\left[l^{\prime}, l^{\prime \prime}\right]\right)-\left[r\left(l^{\prime}\right), r\left(l^{\prime \prime}\right)\right]\right\rangle \\
& =-\left\langle l \otimes l^{\prime} \otimes l^{\prime \prime}, \sum_{\lambda, \mu} \operatorname{sgn}(\lambda) \operatorname{sgn}(\mu)\left(\left[E_{\lambda}, E_{\mu}\right] \otimes E_{-\lambda} \otimes E_{-\mu}+E_{\lambda}\right.\right. \\
& \left.\left.\quad \otimes\left[E_{-\lambda}, E_{\mu}\right] \otimes E_{-\mu}+E_{\lambda} \otimes E_{\mu} \otimes\left[E_{-\lambda}, E_{-\mu}\right]\right)\right\rangle
\end{aligned}
$$

and then split the sum into those terms which are the tensor product of three roots and the remainder. The former can be written as the sum over three roots $\lambda, \mu, \nu$ such that $\lambda+\mu+\nu=0$. Hence [5, Chapter III, Lem. 5.1] applies and leads to this portion of the sum totaling to $\sum_{\lambda+\mu+\nu=0} E_{\lambda} \otimes E_{\mu} \otimes E_{\nu} N_{\mu, \nu}$. The rest is straightforward.)

Hence $\left(g, g^{\star}\right)$ defined with respect to equation (1) is a matched pair of real Lie algebras. The next lemmas compute the necessary details for $g=\operatorname{su}(2)$ which will be needed in the sequel.

LEMMA 2.2. (The example of $g=\operatorname{su}(2)$.) Let $\left\{e_{i}=-l \frac{1}{2} \sigma_{i} ; i=\right.$ $1,2,3\}$ be a basis of $\mathrm{su}(2)$ where

$$
\sigma_{1}=\left(\begin{array}{ll}
0 & 1 \\
1 & 0
\end{array}\right), \quad \sigma_{2}=\left(\begin{array}{cc}
0 & -l \\
l & 0
\end{array}\right), \quad \sigma_{3}=\left(\begin{array}{cc}
1 & 0 \\
0 & -1
\end{array}\right)
$$

are the Pauli spin matrices. Using this basis we shall always identify $\mathrm{su}(2) \cong \mathbf{R}^{3}$ with Lie algebra structure given by the $\mathbf{R}^{3}$ vector product, $\left[\xi, \xi^{\prime}\right]=\xi \times \xi^{\prime}$. For the space $h=\mathrm{su}(2)^{\star}$ we take basis $\left\{f_{i}=-2 l \omega^{i}\right\}$ where $\left\{\omega^{i}\right\}$ denotes the dual basis to the $\left\{e_{i}\right\}$. The basis $\left\{f_{i}\right\}$ will always be used to identify $h \cong \mathbf{R}^{3}$ also. Let the root system for $\mathrm{su}(2)$ be defined 
with choice of Cartan sub-algebra spanned by $\left\{e_{3}\right\}$; then the bracket on $h$ defined by the associated solution of the MCYBE is

$$
\left[l, l^{\prime}\right]=e_{3} \times\left(l \times l^{\prime}\right)
$$

and the actions $\alpha, \beta$ are

$$
\alpha_{\xi}(l)=\xi \times l, \quad \beta_{l}(\xi)=l \times\left(\xi \times e_{3}\right) .
$$

Proof. The Killing form in this basis is $K\left(\xi, \xi^{\prime}\right)=-2 \xi \cdot \xi^{\prime}$ in terms of the Euclidean $\mathbf{R}^{3}$ inner product. There are just two roots of $\operatorname{sl}(2, \mathbf{C})$ which up to normalization are $\sigma_{ \pm}=\frac{1}{2}\left(\sigma_{1} \mp l \sigma_{2}\right)$ and hence

$$
r=\frac{1}{4}\left(\sigma_{+} \otimes \sigma_{-}-\sigma_{-} \otimes \sigma_{+}\right)+t K^{-1}=\frac{1}{4}\left(l \sigma_{1} \otimes \sigma_{2}-l \sigma_{2} \otimes \sigma_{1}\right)+t K^{-1} .
$$

Hence complexifying and using the bases $\left\{e_{i}\right\}$ and $\left\{f_{i}\right\}$ stated to identify $g_{\mathbf{C}}$ and $g_{\mathbf{C}}^{*}$ with $\mathbf{C}^{3}$ we have

$$
r(l)=-l \times e_{3}+t l l .
$$

Now $\alpha_{\xi}(l)_{i}=\sum_{j, k} l_{k} \varepsilon_{k i j} \xi_{j}=(\xi \times l)_{i}$ where $\varepsilon$ is the totally antisymmetric tensor with $\varepsilon_{123}=1$. Hence the bracket on $g_{\mathbf{C}}^{*}$ is

$$
\left[l, l^{\prime}\right]=\left(-l \times e_{3}+t l l\right) \times l^{\prime}+\left(l^{\prime} \times e_{3}+t l l^{\prime}\right) \times l=e_{3} \times\left(l \times l^{\prime}\right)
$$

independent of $t$. Dualizing this gives $\beta$. The structure constants in this basis are real; hence limiting $l, l^{\prime}$ to real values in this basis defines the real Lie algebra $h=g^{\star}$, isomorphic as a vector space to the real dual of $g$, and we also see that $\alpha$ and $\beta$ restrict to give the matched pair $\left(g, g^{\star}\right)$.

LEMMA 2.3. (Description of $H=\mathrm{SU}(2)^{\star}$.) (i) The simply-connected group $H$ of the Lie algebra $h=\operatorname{su}(2)^{\star}$ is the subgroup of $\mathrm{GL}(3, \mathbf{R})$ of the form

$$
\left\{\mathscr{L}=\left(\begin{array}{ccc}
1+L_{3} & 0 & 0 \\
0 & 1+L_{3} & 0 \\
-L_{1} & -L_{2} & 1
\end{array}\right) ; L_{3} \in(-1, \infty), L_{1}, L_{2} \in \mathbf{R}\right\} .
$$

The map $r$ exponentiates to a faithful representation of $H$ as the subgroup of $\mathrm{SL}(2, \mathbf{C})$ defined by matrices

$$
\left\{r(\mathscr{L})=\left(L_{3}+1\right)^{-1 / 2}\left(\begin{array}{cc}
1+L_{3} & 0 \\
L_{1}+\imath L_{2} & 1
\end{array}\right) ; L_{3} \in(-1, \infty), L_{1}+\imath L_{2} \in \mathbf{C}\right\} \text {. }
$$

Either of these identifies the solvable group $H$ with a subspace of $\mathbf{R}^{3}$, namely 3-tuples of the form $\left(L_{1}, L_{2}, L_{3}\right)$ equipped with a non-standard product structure, $L \cdot L^{\prime}=L\left(L_{3}^{\prime}+1\right)+L^{\prime}$. 
(ii) In the representation $r(H)$, the Lie algebra basis vectors of Lemma 2.2 are represented in $\mathrm{sl}(2, \mathrm{C})$ by

$$
r\left(f_{3}\right)=\left(\begin{array}{cc}
\frac{1}{2} & 0 \\
0 & -\frac{1}{2}
\end{array}\right), \quad r\left(f_{1}\right)=\left(\begin{array}{ll}
0 & 0 \\
1 & 0
\end{array}\right), \quad r\left(f_{2}\right)=\left(\begin{array}{ll}
0 & 0 \\
l & 0
\end{array}\right),
$$

and

$$
r(\exp l)=e^{-l_{3} / 2}\left(1+\left(\begin{array}{cc}
l_{3} & 0 \\
l_{1}+l_{2} & 0
\end{array}\right) \frac{e^{l_{3}}-1}{l_{3}}\right) .
$$

(iii) The linear representations $\alpha$ and $\beta$ of $g$ on $h$ and of $h$ on $g$ respectively exponentiate to linear representations of $G$ on $h$ and of $H$ on $g$, according to

$$
\alpha_{x}(l)=\operatorname{Rot}_{x}(l), \quad \beta_{\mathscr{L}}(\xi)=\mathscr{L} \xi .
$$

Here Rot: $G \rightarrow \mathrm{SO}(3)$ and $\mathscr{L}$ is in the defining representation as a subgroup of $\mathrm{GL}(3, \mathbf{R})$, and $l, \xi$ are viewed in $\mathbf{R}^{3}$ as given in Lemma 2.2 .

Proof. We start with part (iii). The exponentiation to $\alpha_{x}(l)=$ Rot is well known. The Lie algebra action $\beta_{l}(\xi)$ given in Lemma 2.2 constitutes a matrix representation of the Lie algebra $h$ as matrices of the form

$$
\left\{(l)=\left(\begin{array}{ccc}
l_{3} & 0 & 0 \\
0 & l_{3} & 0 \\
-l_{1} & -l_{2} & 0
\end{array}\right)\right\} \text {. }
$$

By induction one may compute higher powers of such matrices. In this way one obtains

$$
e^{(l)}=1+\left(\begin{array}{ccc}
l_{3} & 0 & 0 \\
0 & l_{3} & 0 \\
-l_{1} & -l_{2} & 0
\end{array}\right) \frac{e^{l_{3}}-1}{l_{3}} .
$$

This constructs the group $H$ as a group of matrix transformations exponentiating the Lie algebra action $\beta_{l}(\xi)$. Next, using the expression for $r$ given in the proof of Lemma 2.2, with $t=1$, one has $r\left(f_{3}\right)=\imath e_{3}$ and similarly for the others. Exponentiating this matrix representation along the lines above completes part (i) and part (ii).

Note that the basis $\left\{e_{i}\right\}$ for su(2) defined by the Pauli spin matrices in Lemma 2.2 is particularly convenient for exponentiating to an element of $\mathrm{SU}(2)$ and for applications in physics. The corresponding element of $\mathrm{SO}(3)$ is easily obtained as follows. If $x \in \mathrm{SU}(2)$ and $\left(\xi_{1}, \xi_{2}, \xi_{3}\right) \in \mathbf{R}^{3}$ is viewed in $\operatorname{su}(2)$ as $\xi=\sum \xi_{i} e_{i}$, the corresponding action of $\operatorname{Rot}_{x}$ on $\mathbf{R}^{3}$ is given by $\xi \mapsto x \xi x^{-1}$. The identification of 
$\operatorname{su}(2)^{\star}$ with $\left(l_{1}, l_{2}, l_{3}\right) \in \mathbf{R}^{3}$ is chosen so that the action $\alpha$ on $\operatorname{su}(2)^{\star}$ is such a rotation of $\mathbf{R}^{3}$. The physical significance of this is discussed briefly at the end of $\S 4$.

3. Exponentiation to Lie algebra actions of $h$ on $G$ and of $g$ on $H$. In this section we shall compute non-linear actions of $h$ on $G$ and of $g$ on $H$ induced by matched Lie algebra actions $\alpha, \beta$. Indeed, if the corresponding vector fields [7, Chapter I, Prop. 4.1] $\left\{a_{\xi} ; \xi \in g\right\}$ on $H$ and $\left\{b_{l} ; l \in h\right\}$ on $G$ are to later exponentiate to actions of $G$ on $H$ and $H$ on $G$ yielding a matched pair, then they must each obey an equation obtained by suitably differentiating $(*)$. This equation, equation (2) for the $\left\{b_{l}\right\}$, and a similar one for the $\left\{a_{\xi}\right\}$, determines these vector fields completely in terms of the Lie algebra actions $\alpha$ and $\beta$. This is shown in the following theorem.

TheOREM 3.1. Let $(h, g, \alpha, \beta)$ be a matched pair of Lie algebras. Let $G, H$ denote the respective simply-connected groups of $g, h$. We denote also by $\alpha$ (resp. $\beta$ ) the exponentiation of the linear Lie algebra action to a linear action of $G$ on $h$ (resp. $H$ on $g$ ). Then there exists a unique smooth action $b$ of $h$ on $G$ such that

$$
b_{l}(x y)=R_{y^{*}} b_{l}(x)+L_{x^{*}} b_{\alpha_{x-1}(l)}(y), \quad b_{l}(e)=0
$$

where $b_{l} \in T G$ is the vector field corresponding to action by $l \in h, e$ denotes the group identity and $R_{y}$ and $L_{x}$ denote right and left translations in $G$. (Similarly there exists a unique action a of $g$ on $H$ obeying an analogous equation.)

Indeed, given linear actions $\alpha$ on $h$ and $\beta$ on $g$ the vector fields $\left\{b_{l}: l \in h\right\}$ are the unique smooth solutions of

$(* * *) \quad d b_{l} \circ L_{x^{*}} \xi=\left(d\left(R_{*} b_{l}(x)\right)\right) \xi+L_{x^{*}} \beta_{\alpha_{x^{-1}}(l)}(\xi), \quad b_{l}(e)=0$.

(**) for $\beta_{l}\left(\left[\xi, \xi^{\prime}\right]\right)$ is the integrability condition for this. (**) for $\alpha_{\xi}\left(\left[l, l^{\prime}\right]\right)$ is the additional condition that the solutions $\left\{b_{l}\right\}$ are an action of $h$ on all of $G$, i.e.

$$
\left[b_{l}, b_{l^{\prime}}\right]=-b_{\left[l, l^{\prime}\right]}
$$

where the bracket on the left hand side is the Lie bracket in TG. (Similarly for $\left\{a_{\xi}: \xi \in g\right\}$.)

Proof. Differentiate $(*)$ with respect to $\mathscr{L}$ at the identity $e$. This gives the necessary equation (2) where $b_{l}(x)=\left.{ }_{\operatorname{def}} \frac{d}{d t}\right|_{0} \beta_{\exp t l}(x)$ and where we identify $T_{e} H=h$. Differentiate this with respect to $y$ at $y=e$ to give $(* * *) . L_{x^{*}} \xi$ is the left-invariant vector field generated 
by $\xi \in T_{e} G=g$ evaluated at $x$ and $\alpha_{x}(l)$ is the exponential of the linear action $\alpha_{\xi}(l)$. Differentiating again with respect to $x$ and writing the commutator of two left-invariant vector fields in terms of the Lie bracket on $g$, one has on evaluating at $x=e$, the integrability condition $(* *)$ for the derivatives $\left\{\beta_{l}\right\}$ of the $\left\{b_{l}\right\}$ at $x=e$. Here we identified $g=T_{e} G$.

Conversely, write $b_{l}(x) \equiv R_{x^{*}}\left(\bar{B}_{l}(x)\right)$, where $\bar{B}_{l}: G \rightarrow g$. The equation $(* * *)$ in terms of $\bar{B}_{l}$ is

$$
d \bar{B}_{l}=\operatorname{Ad}_{x^{*}} \beta_{\alpha_{x-1}(l)}(\omega) \equiv w_{l} .
$$

Here $\omega$ is the $g$-valued 1-form (Maurer-Cartan form on $G$ ) defined by $\left\langle\omega(x), L_{x^{*}} \xi\right\rangle=\xi$ for all $x \in G, \xi \in g$. Because $G$ is (connected) simply-connected, the equation has a twice-differentiable solution iff the right hand side, $w_{l}$, is closed, and the solution is uniquely determined by $\bar{B}_{l}(e)$. In fact, since $w_{l}$ is smooth, the solution when it exists is smooth. Computing the exterior differential of $w_{l}$,

$$
\begin{aligned}
d w_{l}= & \operatorname{Ad}_{x^{*}} \beta_{\alpha_{x^{-1}}(l)}(d \omega)+\operatorname{Ad}_{x^{*}}\left[\omega, \beta_{\alpha_{x^{-1}}(l)}(\omega)\right] \\
& +\operatorname{Ad}_{x^{*}} \beta_{\alpha_{-\omega}\left(\alpha_{x-1}(l)\right)}(\omega)
\end{aligned}
$$

since $\alpha_{x^{-1}}(l)$ refers to the action exponentiating the linear action $\alpha$. In this expression the exterior multiplication of $g$-valued one-forms is assumed. Using the equations of Maurer and Cartan, $d \omega=-\frac{1}{2}[\omega, \omega]$ we see that $d w_{l}=0$ for all $x \in G, l \in h$ iff $(* *)$ holds for $\beta_{l}$, for all $l^{\prime}=\alpha_{x^{-1}}(l)$. Hence $(* * *)$ has a unique solution iff $(* *)$ holds for $\beta_{l}$ for all $l \in h$. For the resulting $b_{l}$ obeying (***) one may compute that $f(y) \equiv b_{l}(x y)-R_{y^{*}} b_{l}(x)-L_{x^{*}} b_{\alpha_{x-1}(l)}(y)$ as a function of $y$ for each fixed $x$, obeys $d f \circ L_{y^{*}} \xi=d\left(R_{*} f(y)\right) \circ \xi$; hence $f(y)=R_{y^{*}} f(e)$ and so vanishes.

Note that the proof just given can be restated more concisely as follows: Let $\alpha^{*}$ denote the action of $g$ on $h^{*}$ adjoint to $\alpha$. Then $\alpha^{*} \otimes$ ad is an action of $g$ on $h^{*} \otimes g$. The equation $(* *)$ for $\beta_{l}\left(\left[\xi, \xi^{\prime}\right]\right)$ can then be stated concisely as $\beta \in Z_{\alpha^{*} \otimes \text { ad }}^{1}\left(g, h^{*} \otimes g\right)$ (i.e. a 1-cocycle). Because $G$ is (connected) simply-connected, this exponentiates to a 1cocycle on the group, namely, $\bar{B} \in Z_{\alpha^{*} \otimes \mathrm{Ad}}\left(G, h^{*} \otimes g\right)$, cf. equation (2). To actually compute the $\left\{\bar{B}_{l}\right\}$, one must solve a differential equation, as described in detail above.

For the final part of the theorem we write $b_{l}(x) \equiv L_{x^{*}}\left(B_{l}(x)\right)$, and compute

$$
\begin{aligned}
{\left[b_{l}, b_{l^{\prime}}\right](x)=} & L_{x^{*}} \beta_{\alpha_{x^{-1}}\left(l^{\prime}\right)}\left(B_{l}(x)\right)-L_{x^{*}} \beta_{\alpha_{x^{-1}}(l)}\left(B_{l^{\prime}}(x)\right) \\
& +L_{x^{*}}\left[B_{l^{\prime}}(x), B_{l}(x)\right] .
\end{aligned}
$$


For this to equal $-L_{x^{*}} B_{\left[l, l^{\prime}\right]}(x)$ one needs at the identity $x=e$ that $\beta_{l}(\xi)$ is a Lie algebra map. Assuming this and differentiating $L_{x^{-1} *}$ of the above expression with respect to $x$ one has, using $(* * *)$,

$$
\begin{aligned}
& \beta_{\left[\alpha_{x-1}\left(l^{\prime}\right), \alpha_{x-1}(l)\right]}(\xi) \\
& \quad+\beta_{\alpha_{-\xi} \alpha_{x}-1}(l) \\
& \quad\left(B_{l^{\prime}}(x)\right)+\beta_{\alpha_{x-1}\left(l^{\prime}\right)}\left(\left[B_{l}(x), \xi\right]\right)+\left[\left[B_{l^{\prime}}(x), \xi\right]+\beta_{\alpha_{x-1}\left(l^{\prime}\right)}(\xi), B_{l}(x)\right]-l \leftrightarrow l^{\prime} .
\end{aligned}
$$

Meanwhile the differential of $-B_{\left[l, l^{\prime}\right]}(x)$ is, using $(* * *)$ and the assumption that $\beta_{l}(\xi)$ is an action,

$$
\begin{aligned}
{\left[\beta_{\alpha_{x-1}\left(l^{\prime}\right)}\left(B_{l}(x)\right), \xi\right]-\left[\beta_{\alpha_{x-1}(l)}\left(B_{l^{\prime}}(x)\right), \xi\right]-\beta_{\alpha_{x}-1}\left(\left[l, l^{\prime}\right]\right) } & (\xi) \\
& +\left[\left[B_{l^{\prime}}(x), B_{l}(x)\right], \xi\right] .
\end{aligned}
$$

On substituting $(* *)$ and Lemma 3.2 directly below, one finds that these two expressions are equal, i.e. $\left[b_{l}, b_{l^{\prime}}\right]+b_{\left[l, l^{\prime}\right]}$ has zero differential with respect to $x$. Thus, since it vanishes at $x=e$, it vanishes everywhere. The next lemma therefore completes the proof of the theorem.

LEMMA 3.2. Let $(g, h, \alpha, \beta)$ be a matched pair of Lie algebras as in Theorem 3.1 and let $\left\{b_{l}\right\}$ be the resulting global solution of $(* * *)$. Then

$$
\alpha_{x}\left(\left[l, l^{\prime}\right]\right)=\left[\alpha_{x}(l), \alpha_{x}\left(l^{\prime}\right)\right]+\alpha_{B_{l}\left(x^{-1}\right)}\left(\alpha_{x}\left(l^{\prime}\right)\right)-\alpha_{B_{l^{\prime}}\left(x^{-1}\right)}\left(\alpha_{x}(l)\right)
$$

where $b_{l}(x) \equiv L_{x^{*}}\left(B_{l}(x)\right)$.

Proof. Since $\alpha_{x}(l)$ is the exponentiation of the linear action $\alpha$ of $g$ on $h$, the differential of

$$
f(x)=\alpha_{x}\left(\left[l, l^{\prime}\right]\right)-\left(\left[\alpha_{x}(l), \alpha_{x}\left(l^{\prime}\right)\right]+\alpha_{B_{l}\left(x^{-1}\right)}\left(\alpha_{x}\left(l^{\prime}\right)\right)-\alpha_{B_{l^{\prime}}\left(x^{-1}\right)}\left(\alpha_{x}(l)\right)\right)
$$
is

$$
\begin{aligned}
d f \circ L_{x^{*}} \xi= & \alpha_{\xi}\left(\left[l, l^{\prime}\right]\right)-\left[\alpha_{\xi}\left(\alpha_{x}(l)\right), \alpha_{x}\left(l^{\prime}\right)\right]-\left[\alpha_{x}(l), \alpha_{\xi}\left(\alpha_{x}\left(l^{\prime}\right)\right)\right] \\
& -\alpha_{B_{l}\left(x^{-1}\right)}\left(\alpha_{\xi}\left(\alpha_{x}\left(l^{\prime}\right)\right)\right)-\alpha_{\left[\xi, B_{l}\left(x^{-1}\right)\right]+\beta_{-\alpha_{x}(l)}(\xi)}\left(\alpha_{x}\left(l^{\prime}\right)\right) \\
& +\alpha_{B_{l^{\prime}}\left(x^{-1}\right)}\left(\alpha_{\xi}\left(\alpha_{x}(l)\right)\right)+\alpha_{\left[\xi, B_{l^{\prime}}\left(x^{-1}\right)\right]+\beta_{-\alpha_{x}\left(l^{\prime}\right)(\xi)}\left(\alpha_{x}(l)\right) .}
\end{aligned}
$$

By $(* *)$ for $\alpha_{\xi}$, this reduces to $\alpha_{\xi}(f(x))$. Therefore $f(x)=\alpha_{x}(f(e))$ and therefore vanishes. This completes the proof of the lemma, and hence of Theorem 3.1.

It is therefore necessary to solve the differential system $(* * *)$. This can be done either by analytic means or by algebraic methods. 
LEMMA 3.3. (Example of $h=\mathrm{su}(2)^{\star}, G=\mathrm{SU}(2)$.) Using the notation and initial data $\left\{\beta_{l}(\xi)\right\}$ of Lemmas 2.2 and 2.3 , the unique solution of $(* * *)$ on $G=\mathrm{SU}(2)$ is

$$
b_{l}(x)=R_{x^{*}}\left(l \times\left(\alpha_{x}\left(e_{3}\right)-e_{3}\right)\right) .
$$

Proof. An explicit integral formula for the $\left\{b_{l}\right\}$ (resp. $\left\{a_{\xi}\right\}$ ) will arise in the course of proving Theorem 4.2 below. Therefore we shall content ourselves here with merely verifying the solution. For coordinates on $\mathrm{SU}(2)$ we take an embedding in Euclidean space given by a linear representation, the defining one,

$$
x=\left(\begin{array}{ll}
x_{1} & x_{2} \\
x_{3} & x_{4}
\end{array}\right) .
$$

This identifies the fibers of the tangent space also with $M(2, \mathbf{C})$ by $\xi(x)=\xi_{j}^{i} \partial / \partial x_{j}^{i}$ for a vector field $\xi$. In particular, $g \subset M(2, \mathbf{C})$ and $L_{x^{*}} \xi=x \xi, R_{x^{*}} \xi=\xi x$ as matrices. Write $b_{l}(x) \equiv L_{x^{*}} B_{l}(x)$ where $B_{l}: G \rightarrow T_{e} G$. Then $(* * *)$ reads

$$
\left.\frac{d}{d t}\right|_{0} B_{l}(x(1+t \xi))=\left[B_{l}(x), \xi\right]+\left[x^{-1} l x,\left[\xi, e_{3}\right]\right]
$$

where $\alpha_{x^{-1}}(l)=x^{-1} l x$ when $l \in \mathbf{R}^{3}$ is viewed in $\operatorname{su}(2)$ (both $g=\operatorname{su}(2)$ and $h=\operatorname{su}(2)^{\star}$ are identified with this $\mathbf{R}^{3}$ as given in Lemma 2.2). Using the formula $(x \xi)_{j}^{i}\left(\partial / \partial x_{j}^{i}\right) x^{-1}=-\xi x^{-1}$ one can easily verify the solution stated, namely $B_{l}(x)=x^{-1}\left[l, x e_{3} x^{-1}-e_{3}\right] x$, as

$$
d B_{l} \circ x \xi=-\left[\xi, B_{l}(x)\right]+x^{-1}\left[l, x\left[\xi, e_{3}\right] x^{-1}\right] x .
$$

LEMMA 3.4. (Example of $g=\mathrm{su}(2), H=\mathrm{SU}(2)^{\star}$.) Using the notation and initial data $\left\{\alpha_{\xi}(l)\right\}$ of Lemmas 2.2 and 2.3, the unique solution of the equation corresponding to $(* * *)$ for $\left\{a_{\xi}\right\}$ is

$$
a_{\xi}(\mathscr{L})=R_{\mathscr{L}}\left(\xi \times \frac{L}{L_{3}+1}-\frac{1}{2} \xi \times e_{3} \frac{L \cdot L}{\left(L_{3}+1\right)^{2}}\right) .
$$

Proof. For co-ordinates on $H$ we take the defining matrix representation given in Lemma 2.3. For brevity we identify with matrix $\mathscr{L} \in H$, the 3-tuple $L \in \mathbf{R}^{3}$ as given. Writing $a_{\xi}(\mathscr{L})=L_{\mathscr{L}^{*}} A_{\xi}(\mathscr{L})$, where $A_{\xi}: H \rightarrow T_{e} H$, the equation corresponding to (***) becomes

$$
\left.\frac{d}{d t}\right|_{0} A_{\xi}\left(\mathscr{L}\left(1+t l^{\prime}\right)\right)=\left[A_{\xi}(\mathscr{L}), l^{\prime}\right]+\left(\xi+\frac{L \times\left(\xi \times e_{3}\right)}{L_{3}+1}\right) \times l^{\prime}
$$


on substituting the desired $\alpha_{\beta_{\mathscr{L}-1}(\xi)}\left(l^{\prime}\right)$ from Lemmas 2.2 and 2.3. Differentiating the solution stated recovers this. Some useful formulae here are

$$
\left.\frac{d}{d t}\right|_{0} L(t)=L l_{3}^{\prime}+l^{\prime},\left.\quad\left(L_{3}+1\right) \frac{d}{d t}\right|_{0}-\frac{L(t)}{L(t)_{3}+1}=-l^{\prime}
$$

where $L(t)$ is the 3 -tuple corresponding to $\mathscr{L}\left(1+t l^{\prime}\right)$ and where $-L /\left(L_{3}+1\right)$ is the 3 -tuple corresponding to $\mathscr{L}^{-1}$.

4. Exponentiation to a matched pair $(G, H)$. In this section we exponentiate the actions of $h$ on $G$ and $g$ on $H$ to global actions of $H$ on $G$ and $G$ on $H$ respectively. If $\left\{b_{l}\right\}$ are the vector fields on $G$ corresponding to the action of $h$ then the global action, denoted $\tilde{\beta}$, is required to obey

$$
(* * * *) \quad d(\tilde{\beta}(x)) \circ R_{\mathscr{L} *} l^{\prime}=b_{l^{\prime}}\left(\tilde{\beta}_{\mathscr{L}}(x)\right), \quad \tilde{\beta}_{e}(x)=x .
$$

If such global actions integrating the $\{a\}$ and $\{b\}$ exist then they are unique. The following theorems show that if one of $G$ or $H$ is compact (more generally, if the corresponding vector fields are complete) then both the global actions exist and $(G, H)$ are indeed a matched pair of groups. The first lemma shows that it is only necessary to construct one of these actions in order to recover the other.

LEMMA 4.1. Let $(g, h, \alpha, \beta)$ be a matched pair of Lie algebras and $(G, H)$ the simply-connected groups of $(g, h)$. Let $\left\{a_{\xi}\right\},\left\{b_{l}\right\}$ denote the global vector fields corresponding to the induced Lie algebra actions of $g$ on $H$ and $h$ on $G$ respectively. Let $\omega$ denote the left-invariant Maurer-Cartan form on $H$.

(i) If $\tilde{\beta}$ is an action of $H$ on $G$ integrating $\{b\}$ and the family of $h$-valued one-forms

$$
\theta_{x}(\mathscr{L})=\alpha_{\tilde{\beta}_{\mathscr{L}-1}\left(x^{-1}\right)^{-1}}(\omega)
$$

are viewed in the standard way as connections on $H \times H \rightarrow H$, then the expression

$$
\tilde{\alpha}_{x}(\mathscr{L})=P e^{\int_{e}^{\mathscr{L}} \theta_{x}}
$$

is well defined and obeys (*) for $\tilde{\alpha}_{x}(\mathscr{L} \mathscr{M})$. Here Pe $e_{e}^{\int_{e}^{\mathscr{L}} \theta}$ denotes parallel transport by $\theta$ along any piecewise differentiable curve starting at the identity $e$ and ending at $\mathscr{L}$. 
(ii) If $\tilde{\beta}$ obeys $(*)$ for $\tilde{\beta}_{\mathscr{L}}(x y)$ then $\tilde{\alpha}$ is an action integrating $\{a\}$ and hence $(G, H, \tilde{\alpha}, \tilde{\beta})$ is a matched pair of Lie groups.

Proof. (1) $H \times H$ may be regarded as a principal $H$-bundle over $H$. With the standard direct product trivialization, i.e. global section $\sigma: H \rightarrow H \times\{e\}$, the connections on $H \times H$ may be identified with the $h$-valued one-forms on $H$ obtained by pull-back by $\sigma$ [7, Chapter II, Lem. 2.2]. We show first that as such, the connections $\left\{\theta_{x}: x \in G\right\}$ have zero curvature (i.e. obey the Maurer-Cartan equations):

$$
\begin{aligned}
d \theta_{x} & =\alpha_{\tilde{\beta}_{\mathscr{L}^{-1}}\left(x^{-1}\right)^{-1}}\left(-\frac{1}{2}[\omega, \omega]\right)+\alpha_{B_{\omega}\left(\tilde{\beta}_{\mathscr{L}-1}\left(x^{-1}\right)\right)}\left(\alpha_{\tilde{\beta}_{\mathscr{L}^{-1}}\left(x^{-1}\right)^{-1}}(\omega)\right) \\
& =-\frac{1}{2}\left[\theta_{x}, \theta_{x}\right]
\end{aligned}
$$

by Lemma 3.2. This is the geometrical picture of Lemma 3.2 and will prove useful in what follows.

Now in the presence of any connection, each piecewise differentiable curve $\{\gamma(t): t \in[0,1]\}$ in the base $H$ lifts to a curve in the total space $H \times H$, uniquely determined by its starting point in the fiber above $\gamma(0)$ [7, Chapter II, Prop. 3.1]. The operation of parallel transport along $\gamma$ is defined as the map from the fiber above $\gamma(0)$ to the fiber above $\gamma(1)$ that maps the starting point to the endpoint. Since this map commutes with right-translations of the fibers, and the fibers are all identified by $\sigma$, we may consider the map as left translation by an element of $H$, i.e.

$$
P e^{\int_{\gamma} \theta_{x}} \in H
$$

is defined. It is independent of the path of integration since the curvature of each $\theta_{x}$ vanishes and by assumption $\pi_{1}(H)=0$, so that the holonomy group is trivial (cf. [7, Chapter II, Cor. 9.2]). From this point of view, $\tilde{\alpha}_{x}(\mathscr{L})$ is the global section $\tilde{\alpha}_{x}: \mathscr{L} \mapsto\left(\mathscr{L}, \tilde{\alpha}_{x}(\mathscr{L})\right)$ defined in this case by the flat connection $\theta_{x}$.

From a more pedestrian point of view, $\tilde{\alpha}_{x}(t) \equiv P e^{\int_{e}^{\gamma(t) e} \theta_{x}}$ along a given differentiable curve $\gamma$ denotes the unique solution in $H$ of the differential equation

$$
L_{\tilde{\alpha}_{x}(t)^{-1 *}} \frac{d}{d t} \tilde{\alpha}_{x}(t)=\left\langle\theta_{x}(\gamma(t)), \frac{d \gamma}{d t}\right\rangle, \quad \tilde{\alpha}_{x}(0)=e .
$$

That these exist and in our case depend only on the current endpoint $\tilde{\alpha}_{x}(t) \equiv \tilde{\alpha}_{x}(\gamma(t))$, follows from standard results about holonomy groups, as quoted. 
(2) Next we compute

$$
\tilde{\alpha}_{x}(\mathscr{L} \mathscr{M})=P e^{\int_{e}^{\mathscr{L}} \alpha_{\tilde{\beta}_{\mathscr{L}^{\prime}-1}\left(x^{-1}\right)-1}\left(\mathscr{L}^{\prime-1} d \mathscr{L}^{\prime}\right)}
$$

where $\mathscr{L}^{\prime}$ runs along the path of integration and $\mathscr{L}^{\prime-1} d \mathscr{L}^{\prime}$ denotes the Maurer-Cartan form at $\mathscr{L}^{\prime}$. Choose the path of integration to pass through $\mathscr{L}$. At this point let $\mathscr{L}^{\prime \prime} \equiv \mathscr{L}^{-1} \mathscr{L}^{\prime}$. Then $\mathscr{L}^{\prime \prime-1} d \mathscr{L}^{\prime \prime}=$ $\mathscr{L}^{\prime-1} d \mathscr{L}^{\prime}$ and after changing variables in the integration (i.e. in the defining differential equation) we have

$$
\begin{aligned}
\tilde{\alpha}_{x}(\mathscr{L} \mathscr{M}) & =\tilde{\alpha}_{x}(\mathscr{L}) P e^{\int_{e}^{\mathscr{\prime \prime}} \alpha_{\left.\tilde{\beta}_{(\mathscr{L} \mathscr{L}} \prime\right)-1\left(x^{-1}\right)-1\left(\mathscr{L}^{\prime \prime-1} d \mathscr{L}^{\prime \prime}\right)}} \\
& =\tilde{\alpha}_{x}(\mathscr{L}) \tilde{\alpha}_{\tilde{\beta}_{\mathscr{L}-1}\left(x^{-1}\right)^{-1}}(\mathscr{M})
\end{aligned}
$$

since $\tilde{\beta}$ is supposed an action. This completes part (i) of the lemma.

(3) Next suppose that $\tilde{\beta}$ obeys $(*)$. For each $x$ let

$$
f(\mathscr{L})=\tilde{\alpha}_{x^{-1}}\left(\tilde{\alpha}_{x}(\mathscr{L})\right) \text {. }
$$

Then

$$
\begin{aligned}
d f \circ L_{\mathscr{L}^{*}} l^{\prime} & =\left.\frac{d}{d t}\right|_{0} \tilde{\alpha}_{x^{-1}}\left(\tilde{\alpha}_{x}(\mathscr{L}) \tilde{\alpha}_{\tilde{\beta}_{\mathscr{L}-1}\left(x^{-1}\right)^{-1}}\left(\exp t l^{\prime}\right)\right) \\
& =\left.f(\mathscr{L}) \frac{d}{d t}\right|_{0} \tilde{\alpha}_{\tilde{\beta}_{\tilde{\alpha}_{x}(\mathscr{L})^{-1}}(x)^{-1}}\left(\tilde{\alpha}_{\tilde{\beta}_{\mathscr{L}^{-1}}\left(x^{-1}\right)^{-1}}\left(\exp t l^{\prime}\right)\right)
\end{aligned}
$$

by step (2). But $\tilde{\alpha}_{x}(\mathscr{L})$ linearizes to $\alpha_{x}$ about $\mathscr{L}=e$ and the latter is an action while the product $\tilde{\beta}_{\mathscr{L}-1}\left(x^{-1}\right) \tilde{\beta}_{\tilde{\alpha}_{x}(\mathscr{L})^{-1}}(x)=e$ by $(*)$; hence the right hand side is just $d\left(R_{*} f(\mathscr{L})\right) l^{\prime}$. Hence $f(\mathscr{L})=f(e) \mathscr{L}=\mathscr{L}$. Thus

$$
\tilde{\alpha}_{x^{-1}}\left(\tilde{\alpha}_{x}(\mathscr{L})\right)=\mathscr{L} \text {. }
$$

(4) In fact $\tilde{\alpha}$ is an action. For, because $\tilde{\alpha}_{x}$ is a bijection, in particular one may change variables in the path $\mathscr{L}^{\prime}$ defining $\tilde{\alpha}_{x y}(\mathscr{L})$ to $\mathscr{L}^{\prime \prime}=$ $\tilde{\alpha}_{y}\left(\mathscr{L}^{\prime}\right)$. By step (2) we have $\mathscr{L}^{\prime \prime-1} d \mathscr{L}^{\prime \prime}=\alpha_{\tilde{\beta}_{\mathscr{L}^{\prime-1}}\left(y^{-1}\right)^{-1}}\left(\mathscr{L}^{\prime-1} d \mathscr{L}^{\prime}\right)$. This change results in the expression for $\tilde{\alpha}_{x}\left(\tilde{\alpha}_{y}(\mathscr{L})\right)$. (This part also follows from the next theorem.) This completes the proof of the lemma. In fact the hypotheses of part (ii) of the lemma can follow automatically from part (i) leading to the next theorem.

TheOREM 4.2. Let $(g, h, \alpha, \beta)$ be a matched pair of Lie algebras and $G, H$ the simply-connected groups of $g, h$. If $G$ is compact then $(G, H, \tilde{\alpha}, \tilde{\beta})$ is a matched pair of Lie groups, where the actions $\tilde{\alpha}, \tilde{\beta}$ are uniquely determined by $\alpha, \beta$. 
Proof. (1) If the vector fields $\left\{b_{l}: l \in h\right\}$ are all complete, for example if $G$ is compact [7, Chapter I, Prop. 1.6], then the Lie algebra action $b$ of $h$ on $G$ defines a local homomorphism

$$
\tilde{\beta}: H \rightarrow \operatorname{Diff}(G)
$$

(a local homomorphism is a map from a neighborhood of the identity which respects the product of $\mathscr{L}, \mathscr{M}$ whenever $\mathscr{L}, \mathscr{M}, \mathscr{L} \mathscr{M}$ are in the neighborhood). This is because every generator of a one-parameter family in $H$ maps to a generator of a one-parameter family of diffeomorphisms and the generating vector fields $\left\{b_{l}\right\}$ are an involutive distribution (cf. [7, Prop. 1.2]; more specifically, as in Theorem 3.1 we have $\left.\left[b_{l}, b_{l^{\prime}}\right]=-b_{\left[l, l^{\prime}\right]}\right)$. Now every local homomorphism from a simply-connected topological group $H$ to a topological group extends to all of $H$ [2, Chapter II, $\S$ vii, Thm. 3]. Thus $\tilde{\beta}$ is defined as an action of $H$ on $G$ and integrates $b$. Further details of this step can be found in [15, Chapter $3, \S 6$, Cor. 2]. This $\tilde{\beta}$ is uniquely determined by $\{b\}$ since in local co-ordinates in a neighborhood of $(e, x)$ in $H \times G,(* * * *)$ has a unique local solution so that the local homomorphism above is unique. The extension given to all of $H$ is then unique by [2, Chapter II, §vii, Thm. 2].

(2) Because $\tilde{\beta}$ is an action, part (i) of Lemma 4.1 applies, i.e. $\tilde{\alpha}$ is well-defined. Let

$$
\begin{gathered}
\psi_{\mathscr{L}}(x ; \xi)=\beta_{\tilde{\alpha}_{x^{-1}}\left(\mathscr{L}^{-1}\right)^{-1}}(\xi) \\
G(\mathscr{L}, x ; \xi)=\left.L_{\tilde{\beta}_{\mathscr{L}}(x)^{-1} *} \frac{d}{d t}\right|_{0} \tilde{\beta}_{\mathscr{L}}(x(\exp t \xi))-\psi_{\mathscr{L}}(x ; \xi) .
\end{gathered}
$$

We shall show that $G$ vanishes. Thus we compute

$$
\begin{aligned}
d G(\mathscr{L}, x ; \xi) \circ R_{\mathscr{L}^{*}} l^{\prime}= & -\left[B_{l^{\prime}}\left(\tilde{\beta}_{\mathscr{L}}(x)\right), G(\mathscr{L}, x ; \xi)+\psi_{\mathscr{L}}(x ; \xi)\right] \\
& +d B_{l^{\prime}}\left(\tilde{\beta}_{\mathscr{L}}(x)\right)\left(G(\mathscr{L}, x ; \xi)+\psi_{\mathscr{L}}(x ; \xi)\right) \\
& -\beta_{\theta_{x^{-1}}\left(\mathscr{L}^{-1} ; l^{\prime}\right)}\left(\beta_{\tilde{\alpha}_{x^{-1}}(\mathscr{L}-1)^{-1}}(\xi)\right)
\end{aligned}
$$

where $b_{l^{\prime}}(x) \equiv L_{x^{*}}\left(B_{l^{\prime}}(x)\right)$ and where $\left\langle\theta_{x}, L_{\mathscr{L}^{*}} l^{\prime}\right\rangle \equiv \theta_{x}\left(\mathscr{L} ; l^{\prime}\right)$ was used according to equation (3). Substituting the equation $(* * *)$ for $d B$, we obtain

$$
d G(\mathscr{L}, x ; \xi) \circ R_{\mathscr{L}^{*}} l^{\prime}=\beta_{\theta_{x^{-1}}\left(\mathscr{L}^{-1} ; l^{\prime}\right)}(G(\mathscr{L}, x ; \xi))
$$

Hence because $G(e, x ; \xi)=0$ and $G$ obeys a first order linear equation, we have that $G$ vanishes identically.

(3) Next we compute $d(\tilde{\alpha}(\mathscr{L})) \circ R_{x^{*}} \xi \equiv \bar{a}_{\xi}\left(\tilde{\alpha}_{x}(\mathscr{L})\right)$. In the definition of $\tilde{\alpha}$ let the path $\mathscr{L}^{\prime}(t)$ be $\mathscr{L}^{\prime}(0)=e, \mathscr{L}^{\prime}(1) \equiv \mathscr{L}$. For clarity we also 
denote left and right translations by juxtaposition. Then

$$
\begin{aligned}
\bar{a}_{\xi}\left(\tilde{\alpha}_{x}(\mathscr{L})\right) \\
\quad=\left.\int_{0}^{1} d t \tilde{\alpha}_{x}\left(\mathscr{L}^{\prime}(t)\right) \frac{d}{d s}\right|_{0} \theta_{(\exp s \xi) x}\left(\mathscr{L}^{\prime}(t) ; \mathscr{L}^{\prime}(t)^{-1} \dot{\mathscr{L}}^{\prime}(t)\right) P e^{\int_{\mathscr{L}^{\prime}(t)}^{\mathscr{L}} \theta_{x}} \\
\quad=\int_{e}^{\mathscr{L}} \tilde{\alpha}_{x}\left(\mathscr{L}^{\prime}\right) \alpha_{\psi_{\mathscr{L}^{\prime}-1}\left(x^{-1} ; \xi\right)}\left(\theta_{x}\right) \tilde{\alpha}_{x}\left(\mathscr{L}^{\prime}\right)^{-1} \tilde{\alpha}_{x}(\mathscr{L}) \\
\quad=\left(\int_{e}^{\tilde{\alpha}_{x}(\mathscr{L})} \operatorname{Ad}_{\left.\mathscr{L}^{\prime \prime} * \alpha_{\beta_{\mathscr{L}^{\prime \prime}-1}(\xi)}\left(\mathscr{L}^{\prime \prime-1} d \mathscr{L}^{\prime \prime}\right)\right) \tilde{\alpha}_{x}(\mathscr{L})}\right.
\end{aligned}
$$

using step (2) and that $\alpha$ is an action, and then changing variables in the integral to $\mathscr{L}^{\prime \prime}=\tilde{\alpha}_{x}\left(\mathscr{L}^{\prime}\right)$. Here the associated change in integration measure is $\mathscr{L}^{\prime \prime-1} d \mathscr{L}^{\prime \prime}=\alpha_{\tilde{\beta}_{\mathscr{L}^{\prime}-1}\left(y^{-1}\right)^{-1}}\left(\mathscr{L}^{\prime-1} d \mathscr{L}^{\prime}\right)\left(\right.$ since $\alpha_{x}: h \rightarrow h$ is an action, this is non-singular.)

Setting $x=e$, and differentiating with respect to $\mathscr{L}$ we see that $\bar{a}_{\xi}$ obeys the equation corresponding to $(* * *)$ for $a_{\xi}$; hence by the uniqueness in Theorem 3.1 we have that $\bar{a}=a$. Hence $\tilde{\alpha}$ integrates $a$ in the equation corresponding to $(* * * *)$. Since $\tilde{\alpha}$ is globally defined, the vector fields $\left\{a_{\xi}\right\}$ are therefore complete. Hence $a$ defines a local homomorphism which is $\tilde{\alpha}$ from $G$ to $\operatorname{Diff}(H)$. Hence as in step (1), $\tilde{\alpha}$ extends to all of $G$, i.e. is an action, integrating $a$.

(4) By Lemma 4.1(ii) applied to the action $\tilde{\alpha}$, we have that

$$
\tilde{\beta}_{\mathscr{L}}(x)=P e^{\int_{e}^{x} \psi_{\mathscr{L}}}
$$

where $\psi_{\mathscr{L}}=\psi_{\mathscr{L}}(\omega)$ and $\omega$ is the left invariant Maurer-Cartan form on $G$. This is because by the lemma the right hand side is an action integrating $b$ and is therefore equal to $\tilde{\beta}$ by uniqueness of $\tilde{\beta}$ in step (1). But this exhibits $\tilde{\beta}$ as obeying (*) by Lemma 4.1(i). Thus $(G, H, \tilde{\alpha}, \tilde{\beta})$ is a matched pair integrating $(a, b)$. These were in turn uniquely determined by $(\alpha, \beta)$. This concludes the proof of the theorem.

Note that compactness is sufficient but not necessary-we have just proven that whenever one of the induced Lie algebra actions $\{a\}$ or $\{b\}$ exponentiates to a global action then so does the other, and the result is indeed a matched pair. We now compute as an example the case $G=\mathrm{SU}(2), H=\mathrm{SU}(2)^{\star}$.

THEOREM 4.3. ( $A$ solution of the compatability conditions $(*)$ for $G=\mathrm{SU}(2)$ and $H=\mathrm{SU}(2)^{\star}$.) The Lie algebra actions of Lemma 2.2 exponentiate via Lemmas 3.3 and 3.4 to actions of $G$ on $H$ and of $H$ 
on $G$ given explicitly by

(i)

$$
\tilde{\beta}_{\mathscr{L}}(x)=\frac{r(\mathscr{L}) x\left(\begin{array}{ll}
0 & 0 \\
0 & 1
\end{array}\right)+r(\mathscr{L})^{-\dagger} x\left(\begin{array}{ll}
1 & 0 \\
0 & 0
\end{array}\right)}{\left|r(\mathscr{L}) x\left(\begin{array}{ll}
0 & 0 \\
0 & 1
\end{array}\right)\right|_{2}}
$$

where $r^{-\dagger}$ is the conjugate gradient representation of the $r$ given in Lemma 2.3, and ||$_{2}$ denotes the matrix norm in $\operatorname{SL}(2, \mathrm{C})$. Note that $P: \mathrm{SL}(2, \mathrm{C}) \rightarrow \mathrm{SU}(2)$ defined by

$$
P(x)=\frac{x\left(\begin{array}{ll}
0 & 0 \\
0 & 1
\end{array}\right)+x^{-\dagger}\left(\begin{array}{ll}
1 & 0 \\
0 & 0
\end{array}\right)}{\left|x\left(\begin{array}{ll}
0 & 0 \\
0 & 1
\end{array}\right)\right|_{2}}
$$

is a projection map. In terms of it, $\tilde{\beta}_{\mathscr{L}}(x)=P(r(\mathscr{L}) x)$.

(ii)

$$
\tilde{\alpha}_{x}(\mathscr{L})=\frac{1}{2} \frac{L \cdot L}{L_{3}+1} e_{3}+\operatorname{Rot}_{x}\left(L-\frac{1}{2} \frac{L \cdot L}{L_{3}+1} e_{3}\right)
$$

where $H$ is identified with a subspace of $\mathbf{R}^{3}$ and Rot is the rotation of $\mathbf{R}^{3}$, as given in Lemma 2.3. Thus $\tilde{\alpha}$ is a rotation of the 3-plane $\left\{L \in \mathbf{R}^{3} ; L_{3}>-1\right\}$ about a point dependent on the argument. These actions render $\left(\mathrm{SU}(2), \mathrm{SU}(2)^{\star}\right)$ a matched pair.

Proof. (i) In our standard matrix co-ordinates for $\operatorname{SL}(2, \mathrm{C}), x=$ $\left(\begin{array}{ll}x_{1} & x_{2} \\ x_{3} & x_{4}\end{array}\right)$, we have to solve $\dot{x}=\left[l, x e_{3} x^{-1}-e_{3}\right] x$ where

$$
l=-l \frac{1}{2}\left(\begin{array}{cc}
l_{3} & l_{1}-l_{2} \\
l_{1}+l l_{2} & -l_{3}
\end{array}\right)
$$

is the vector $l$ viewed in $\operatorname{su}(2)$. Writing $\tilde{l}=l_{1}+l_{2} \in \mathbf{C}$ the equation becomes

$$
\dot{x}=-\frac{1}{2}\left(\begin{array}{cc}
\overline{\tilde{l}}\left(x_{3} x_{4} x_{1}-2 x_{2} x_{3}^{2}\right)+\tilde{l} x_{1}^{2} x_{2}-2 l_{3} x_{1} x_{2} x_{3} & \overline{\tilde{l}}\left(-x_{2} x_{3} x_{4}\right)+\tilde{l} x_{1} x_{2}^{2}-2 l_{3} x_{1} x_{2} x_{4} \\
\tilde{l}\left(x_{1} x_{2} x_{3}\right)-\tilde{l} x_{3}^{2} x_{4}-2 l_{3} x_{1} x_{3} x_{4} & \tilde{l}\left(2 x_{2}^{2} x_{3}-x_{1} x_{2} x_{4}\right)+\tilde{\widetilde{l}}\left(-x_{3} x_{4}^{2}\right)-2 l_{3} x_{3} x_{4} x_{2}
\end{array}\right) .
$$

From this one may compute

$$
\begin{gathered}
x_{2} \dot{x}_{4}-x_{4} \dot{x}_{2}=l_{3}\left(-x_{2} x_{4}\right)+\tilde{l} x_{2}^{2}, \\
x_{3} \dot{x}_{1}-x_{1} \dot{x}_{3}=l_{3}\left(-x_{1} x_{3}\right)-\tilde{\widetilde{l}} x_{3}^{2}, \quad x_{3} \dot{x}_{2}-x_{2} \dot{x}_{3}=0
\end{gathered}
$$

(Since we are interested in the case $x \in \mathrm{SU}(2)$, which is preserved by the differential equation, we know that $x_{1}=\bar{x}_{4}, x_{2}=-\bar{x}_{3}$.) For 
$x \in \mathrm{SU}(2)$, if $x_{3}(0)=0$ or $x_{2}(0)=0$ then both vanish and hence by inspection $\dot{x}=0$ so $x=x(0)$. Otherwise we therefore have

$$
\frac{x_{4}}{x_{2}}=\frac{x_{4}(0)}{x_{2}(0)} e^{-l_{3} t}-\tilde{l} \frac{e^{-l_{3} t}-1}{l_{3}}, \frac{x_{3}}{x_{2}}=\frac{x_{3}(0)}{x_{2}(0)} .
$$

Evaluating at $t=1$ and recalling that $\mathscr{L}=\exp l$ is described by the 3-tuple $L=l\left(e^{l_{3}}-1\right) / l_{3}$, we solve for $-x_{2} x_{3}=\left|x_{3}\right|^{2}$ to obtain $x_{3}$ and hence the other components. The result for initial data $x$ is

$$
\tilde{\beta}_{\mathscr{L}}\left(\left(\begin{array}{ll}
x_{1} & x_{2} \\
x_{3} & x_{4}
\end{array}\right)\right)=\frac{\left(\begin{array}{cc}
x_{1}-\left(L_{1}-\imath L_{2}\right) x_{3} & x_{2}\left(L_{3}+1\right) \\
x_{3}\left(L_{3}+1\right) & x_{4}+\left(L_{1}+\imath L_{2}\right) x_{2}
\end{array}\right)}{\sqrt{\left|x_{2}\right|^{2}\left(L_{3}+1\right)^{2}+\left|x_{4}+\left(L_{1}+l L_{2}\right) x_{2}\right|^{2}}} .
$$

This can be written in the form stated in the theorem. The map $P$ conveniently maps $x \in \operatorname{SL}(2, \mathbf{C})$ to

$$
\frac{\left(\begin{array}{cc}
\bar{x}_{4} & x_{2} \\
-\bar{x}_{2} & x_{4}
\end{array}\right)}{\sqrt{\left|x_{2}\right|^{2}+\left|x_{4}\right|^{2}}} \in \mathrm{SU}(2)
$$

This completes the derivation of part (i).

(ii) In our standard co-ordinates we have to solve

$$
\dot{L}=\xi \times\left(L-\frac{1}{2} \frac{L \cdot L}{L_{3}+1} e_{3}\right)
$$

First note that then $L: L=2 L \cdot \dot{L}=(\xi \times L) \cdot e_{3} L \cdot L /\left(L_{3}+1\right)$ while $\dot{L}_{3}=e_{3} \cdot(\xi \times L)$. Hence the expression $L \cdot L /\left(L_{3}+1\right)$ is an invariant of the motion, hence is equal to the initial value. Substituting this in the equation to be solved immediately gives the solution. $L \cdot \xi$ is also an invariant.

(One may also check directly that these expressions are indeed actions. It is rather hard to check directly that they fulfill $(*)$ as they must by construction. However, this too has been verified by extensive computations using the symbol manipulation package SMP.) These actions are not free.

Proposition 4.4. (i) The fixed points of the action $\tilde{\beta}$ of $H$ on $G$ are the diagonal subgroup $\left\{\left(\begin{array}{cc}e^{t \theta} & 0 \\ 0 & e^{-t \theta}\end{array}\right) ; \theta \in[0,2 \pi]\right\}$.

(ii) The fixed points in $H$ of the action $\tilde{\alpha}_{\exp t \xi}$ of the subgroup of $G$ generated by $\xi$ are the line $\left\{L_{\lambda} ; \lambda \in \mathbf{R}\right\}$ in $\mathbf{R}^{3}$ defined by $(-1, \infty)$ along the $e_{3}$-axis in the case $\xi \propto e_{3}$ and otherwise by

$$
L_{\lambda}(\xi)=\left(\frac{\lambda}{z} \xi_{1}, \frac{\lambda}{z} \xi_{2}, z-1\right)
$$


where

$$
z=\sqrt{\frac{1}{2}+\lambda \xi_{3}+\sqrt{\frac{1}{4}+\lambda \xi_{3}+\lambda^{2} \xi \cdot \xi}} .
$$

The line passes through the origin where its tangent is $\xi$. The orbits of the action are nonconcentric nested spheres in $\mathbf{R}^{3}$ of center $(0,0, h): h>$ 0 and radius $\sqrt{h(h+2)}$. Thus the action of $\mathrm{SU}(2)$ on $H$ is qualitatively a non-linear distortion of the standard rotation of $\mathbf{R}^{3}$ with $L_{\lambda}(\xi)$ playing the role of axis of rotation.

Proof. (i) is evident from the co-ordinate expression for $\tilde{\beta}_{\mathscr{L}}(x)$ given in the proof of Theorem 4.3. For (ii) we seek $L$ in the subspace of $\mathbf{R}^{3} ; L_{3}>-1$ such that $L\left(L_{3}+1\right)-\frac{1}{2} L \cdot L e_{3}=\lambda \xi$ for some $\lambda \in \mathbf{R}$. Writing $z=L_{3}+1$ the equation for $L_{1}, L_{2}$ is as shown and in the $e_{3}$ component it is $z^{2}\left(z^{2}-1\right)-2 z^{2} \lambda \xi_{3}=\lambda^{2}\left(\xi_{1}^{2}+\xi_{2}^{2}\right)$.

In conclusion, putting together $\S \S 2-4$ of this paper we have two corollaries of Theorem 4.2 and its proof.

CoROllaRY 4.5. Let $G$ be a semi-simple simply-connected Lie group. Let $r$ be a solution of the MCYBE on $g_{\mathrm{C}}$ the complexification of $g$ the Lie algebra of $G$ such that there is a real form $g^{\star}$ of $g_{\mathrm{C}}^{*}$ and such that the mutual co-adjoint actions of $g_{\mathrm{C}}$ and $g_{\mathrm{C}}^{*}$ restrict to $g$ and $g^{\star}$. If the vector fields of $g^{\star}$ acting on $G$ are complete then $\left(G, G^{\star}\right)$ is a matched pair. Here $G^{\star}$ is the simply-connected group of $g^{\star}$.

We saw in Proposition 2.1 that when $G$ is compact, a suitable $r$ and $g^{\star}$ exist. We also note some elementary properties of the $G^{\star}$ that results for this particular $r$ :

COROLlARY 4.6. Let $G$ be a compact semi-simple simply-connected Lie group. Then there exists a simply-connected Lie group $G^{\star}$ of the same dimension as $G$, such that $\left(G, G^{\star}\right)$ is a matched pair.

Proposition 4.7. Let $G$ be a compact semisimple Lie group and $G^{\star}$ the matching group defined with respect to the solution of the MCYBE stated in equation (1). Then $G^{\star}$ is non-compact and solvable.

Proof. This is an elementary computation from the definitions of the bracket in $g^{\star}$ and $g_{\mathrm{C}}^{*}$ defined by the solution of the MCYBE stated in equation (1). Details are omitted in view of the following connection with the Iwasawa decomposition (see the note added at the end of the paper). The bicrossproduct group $G \bowtie G^{\star}$ for this solution of 
the MCYBE can be identified with the simply-connected group of $g_{\mathrm{C}}$ considered as a Lie algebra over $\mathbf{R}$. The groups $G$ and $G^{\star}$ can then be identified respectively with the compact and solvable groups in the Iwasawa decomposition of this.

Note that one may also expect that a Lie algebra matched pair exponentiates to a matched pair of groups in somewhat greater generality than that stated in Corollary 4.5. The question of exponentiation of Lie algebra actions on manifolds was studied in [15, Chapter 3 and 4], where somewhat weaker conditions than compactness are formulated. As remarked after the proof of Theorem 4.2, any time one of the Lie algebra actions exponentiates we have a matched pair.

As an example we carried out the construction in detail in the case $G=\mathrm{SU}(2)$. The problem is interesting also in the non-simple and noncompact case. An example is the case $G=\mathbf{R}, H=\mathbf{R}$ studied in [12, Lemma 2.6] where $(*)$ was solved completely. For even in this case the solutions did exist, on a restricted domain, yielding bicrossproduct semigroups $\mathbf{R} \bowtie \mathbf{R}_{\geq 0}$. Further work will include attempting the construction for each of the classical Lie groups (not just the compact ones) and giving details of the associated bicrossproduct Hopfvon Neumann algebras. These latter are interesting from the point of view of non-commutative geometry and also from an interpretation as the algebra of observables of a quantum system [13].

Note that in this physical picture the generator of translations in a space is called momentum, for example "angular momentum" in reference to the action of $\mathbf{S U}(2)$ on $\mathbf{R}^{3}$. What the construction of a matched pair achieves is to modify the action of the momentum group in such a way that the space that was acted upon can act back on the space that was originally acting, in keeping with an interesting physical principle of the German philosopher E. Mach [10, Chapter 2. v-vii]. Mach sought to generalize Newton's third law in mechanics ("every action has an equal and opposite reaction") to a fundamental philosophical principle of reciprocity in the actions of particles on the motion of other particles, and vice versa. (These ideas led directly to Einstein's formulation of gravity.) It is only in modern times, with the advent of quantum mechanics, that particle motion is expressed in the simplest cases through group theory and algebra. Indeed, the author's original motivation for the present paper comes as part of an attempt [13] [14] to update Mach's ideas in a quantum mechanical setting, see $[12, \S 1.1]$ for a discussion. This physical motivation for 
bicrossproducts is in addition to the independent physical motivations underlying [4] [17] [1] [6].

Note added. After this work was submitted for publication, there appeared a new preprint [9] in which the basic properties of matched pairs of groups and some of the results here, are developed independently. I would like to thank M. A. Rieffel for sending me a copy of this. M. A. Rieffel also alerted us both to reference [11] where some basic properties of matched pairs of groups were noted somewhat previously. Corollary 4.6 and Proposition 4.7, obtained as an application of our constructive method in the case of the specific solution (1) of the CYBE, are deduced from the Iwasawa decomposition in the approach of [11] and [9]. Part of Theorem 3.1 also appears to have been obtained independently in the preprint [8].

\section{REFERENCES}

[1] A. Belavin and V. G. Drinfel'd, Triangle equations and simple Lie algebras, Sov. Sci. Reviews Section, C4 (1984), 93-165.

[2] C. Chevalley, Theory of Lie Groups, PUP (1946).

[3] A. Connes, $C^{*}$ algèbres et géométrie différentielle, C. R. Acad. Sci. Paris, 290 (1980), 599-604.

[4] V. G. Drinfel'd, Hamiltonian structures on Lie groups, Lie bialgebras and the geometric meaning of the classical Yang-Baxter equations, Sov. Math. Dokl., 27 (1983), 68.

[5] S. Helgason, Differential Geometry, Lie Groups and Symmetric Spaces, Academic Press, (1978).

[6] M. Jimbo, Quantum $R$ matrix related to generalized Toda system: an algebraic approach, Springer Lecture Notes in Physics, 246 (1986).

[7] S. Kobayashi and N. Nomizu, Foundations of Differential Geometry, J. Wiley and Sons, (1963) Volume 1.

[8] Y. Kossmann-Schwartzbach and F. Magri, Poisson-Lie groups and complete integrability, Publ IRMA Lille, (1987).

[9] J.-H. Lu, and A. Weinstein, Poisson Lie groups, dressing transformations and Bruhat decompositions, Berkeley math preprint (1988).

[10] E. Mach, The Science of Mechanics, (1893) transl. T. J. McCormack, Open Court Publ. (1960).

[11] G. W. Mackey, Products of subgroups and projective multipliers, Colloquia Mathematical Societas, Janos Bolyais, 5, Hilbert Space Operators, Tihany (Hungary) (1970).

[12] S. Majid, Physics for algebraists: non-commutative and non-cocommutative Hopf algebras by a bicrossproduct construction, (1987), to appear J. Algebra, 128 (1990).

[13] _ Hopf algebras for physics at the Planck scale, J. Classical and Quantum Gravity, 5 (1988), 1587-1606.

[14] _ Hopf-von Neumann algebra bicrossproducts, Kac algebra bicrossproducts, and classical Yang-Baxter equations, (1988), to appear J. Funct. Anal. 
[15] R. Palais, Global formulation of the Lie theory of transformation groups, Memoirs Amer. Math. Soc., 23 (1957), 1-123.

[16] M. A. Rieffel, Projective modules over higher dimensional non-commutative tori, Canad. J. Math., XL, No. 2 (1988), 257-338.

[17] M. A. Semenov-Tyan-Shanskii, What is a classical R-matrix?, Funktional. Anal. i Prilozhen., 17 (1983), 17-33.

[18] M. Takeuchi, Matched pairs of groups and bismash products of Hopf algebras, Comm. Algebra, 9 (1981), 841-882.

Received March 4, 1988 and in revised form December 6, 1988. Work supported in part by the Herchel Smith Scholarship of Emmanuel College, Cambridge, England.

HARVARD UNIVERSITY

CAMBridge, MA 02138 


\section{PACIFIC JOURNAL OF MATHEMATICS EDITORS}

\author{
V. S. VARADARAJAN \\ (Managing Editor) \\ University of California \\ Los Angeles, CA 90024-1555-05 \\ Herbert Clemens \\ University of Utah \\ Salt Lake City, UT 84112 \\ THOMAs ENRIGHT \\ University of California, San Diego \\ La Jolla, CA 92093
}

R. FINN

Stanford University

Stanford, CA 94305

HermanN FlaschKa

University of Arizona

Tucson, AZ 85721

VAughan F. R. Jones

University of California

Berkeley, CA 94720

STEVEN KeRCKHOFF

Stanford University

Stanford, CA 94305

\author{
RobION KIRBY \\ University of California \\ Berkeley, CA 94720 \\ C. C. MoOre \\ University of California \\ Berkeley, CA 94720
}

Harold Stark

University of California, San Diego

La Jolla, CA 92093

\section{ASSOCIATE EDITORS
R. ARENS
E. F. BECKENBACH
B. H. NeumanN
F. WoLF
K. YoshidA (1906-1982)}

\section{SUPPORTING INSTITUTIONS}

UNIVERSITY OF ARIZONA
UNIVERSITY OF BRITISH COLUMBIA
CALIFORNIA INSTITUTE OF TECHNOLOGY
UNIVERSITY OF CALIFORNIA
MONTANA STATE UNIVERSITY
UNIVERSITY OF NEVADA, RENO
NEW MEXICO STATE UNIVERSITY
OREGON STATE UNIVERSITY

\author{
UNIVERSITY OF OREGON \\ UNIVERSITY OF SOUTHERN CALIFORNIA \\ STANFORD UNIVERSITY \\ UNIVERSITY OF HAWAII \\ UNIVERSITY OF TOKYO \\ UNIVERSITY OF UTAH \\ WASHINGTON STATE UNIVERSITY \\ UNIVERSITY OF WASHINGTON
}

The Supporting Institutions listed above contribute to the cost of publication of this Journal, but they are not owners or publishers and have no responsibility for its content or policies.

Mathematical papers intended for publication in the Pacific Journal of Mathematics should be in typed form or offset-reproduced (not dittoed), double spaced with large margins. Please do not use built up fractions in the text of the manuscript. However, you may use them in the displayed equations. Underline Greek letters in red, German in green, and script in blue. The first paragraph must be capable of being used separately as a synopsis of the entire paper. In particular it should contain no bibliographic references. Please propose a heading for the odd numbered pages of less than 35 characters. Manuscripts, in triplicate, may be sent to any one of the editors. Please classify according to the scheme of Math. Reviews, Index to Vol. 39. Supply name and address of author to whom proofs should be sent. All other communications should be addressed to the managing editor, or Elaine Barth, University of California, Los Angeles, California 90024-1555-05.

There are page-charges associated with articles appearing in the Pacific Journal of Mathematics. These charges are expected to be paid by the author's University, Government Agency or Company. If the author or authors do not have access to such Institutional support these charges are waived. Single authors will receive 50 free reprints; joint authors will receive a total of 100 free reprints. Additional copies may be obtained at cost in multiples of 50 .

The Pacific Journal of Mathematics is issued monthly as of January 1966. Regular subscription rate: $\$ 190.00$ a year (5 Vols., 10 issues). Special rate: $\$ 95.00$ a year to individual members of supporting institutions.

Subscriptions, orders for numbers issued in the last three calendar years, and changes of address should be sent to Pacific Journal of Mathematics, P.O. Box 969, Carmel Valley, CA 93924, U.S.A. Old back numbers obtainable from Kraus Periodicals Co., Route 100, Millwood, NY 10546.

The Pacific Journal of Mathematics at P.O. Box 969, Carmel Valley, CA 93924 (ISSN 0030-8730) publishes 5 volumes per year. Application to mail at Second-class postage rates is pending at Carmel Valley, California, and additional mailing offices. Postmaster: send address changes to Pacific Journal of Mathematics, P.O. Box 969, Carmel Valley, CA 93924.

\section{PUBLISHED BY PACIFIC JOURNAL OF MATHEMATICS, A NON-PROFIT CORPORATION}

Copyright (C) 1990 by Pacific Journal of Mathematics 


\section{Pacific Journal of Mathematics}

\section{Vol. 141, No. 2 December, 1990}

Ulrich F. Albrecht, Locally $A$-projective abelian groups and

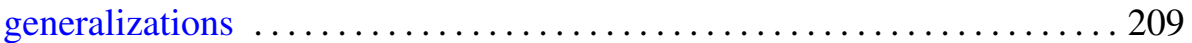

Michel Carpentier, Sommes exponentielles dont la géométrie est très belle: $p$-adic estimates ..................................... 229

G. Deferrari, Angel Rafael Larotonda and Ignacio Zalduendo, Sheaves and functional calculus

Jane M. Hawkins, Properties of ergodic flows associated to product odometers ........................................287

Anthony To-Ming Lau and Viktor Losert, Complementation of certain subspaces of $L_{\infty}(G)$ of a locally compact group ............... 295

Shahn Majid, Matched pairs of Lie groups associated to solutions of the

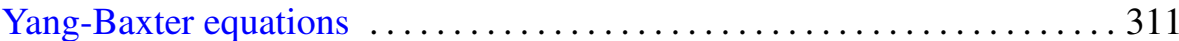

Diego Mejia and C. David (Carl) Minda, Hyperbolic geometry in $k$-convex

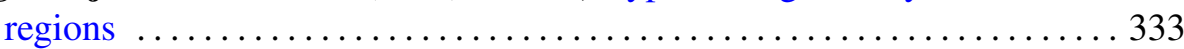

Vladimír Müller, Kaplansky's theorem and Banach PI-algebras ...........355

Raimo Näkki, Conformal cluster sets and boundary cluster sets coincide . . . 363

Tomasz Przebinda, The wave front set and the asymptotic support for $p$-adic groups

R. F. Thomas, Some fundamental properties of continuous functions and topological entropy 